\title{
Avaliação de Ambientes Educacionais: Estudos de Caso com Sim-Colmeia e Educ-MAS GA
}

\author{
Alane de Almeida Ferreira, Rosa Maria Moreira da Costa, Vera Maria Benjamim \\ Werneck \\ Programa de Mestrado em Ciências Computacionais \\ Universidade do Estado do Rio de Janeiro (UERJ) \\ Rua São Francisco Xavier, 524 - $6^{\circ}$ Andar, Maracanã, 20.550-013, Rio de Janeiro-RJ, \\ Brasil \\ Alane.ferreira.af@hotmail.com, \{rcosta, vera\}@ime.uerj.br
}

\begin{abstract}
This paper describes learning evaluation models that are being proposed aiming at observing learning support evaluation process. The learning environments Educ-MAS GA (territory of Analytics Geometry) and Sim-Colmeia (simulation of the activities of a hive in Biology instruction) were developed under the scope of Educational Virtual Environments project. The paper shows an offer of valuation from these Educational Virtual Environments for the purpose of analyze the students learning process. This process is being applied in Analytics Geometry instruction with Educ-MAS GA with students from Technology graduation and with Sim-Colmeia with Biology highschool students. As of these experiments, it will be possible to validate these Educational Environments and analyze the learning process, like the students and teachers satisfaction using the Learning Environments.
\end{abstract}

Resumo. Modelos de avaliação de aprendizagem estão sendo propostos atualmente com o objetivo de observar o processo de avaliação de ambientes educacionais. Os ambientes de aprendizagens Educ-MAS GA (domínio da Geometria Analítica) e Sim-Colmeia (simulação das atividades de uma colmeia no ensino de Biologia) foram desenvolvidos no âmbito do projeto de Ambientes Virtuais Educacionais. Este trabalho apresenta uma proposta de avaliação de ambientes educacionais com o intuito de verificar o processo de aprendizado dos alunos. Este processo está sendo experimentado no ensino de Geometria Analítica com Educ-MAS GA para alunos da Graduação da área Tecnológica e de Biologia para ensino Médio com Sim-Colmeia. A partir desses experimentos poderemos validar esses ambientes educacionais e verificar o processo de aprendizagem, como a satisfação dos alunos e professores usando ambientes de aprendizado.

\section{Introdução}

O uso da tecnologia no contexto da educação tem crescido ultimamente. Ela tem estimulado a criação de ferramentas que auxiliam no processo de aprendizagem, onde não somente a sala de aula é capaz de gerar conhecimento. Com a era da informatização, houve um crescimento de ambientes educacionais que vem se destacando ao longo dos anos por sua capacidade de proporcionar experiências educativas aos alunos. 


\section{CBIE-LACLO 2015}

Anais dos Workshops do IV Congresso Brasileiro de Informática na Educação (CBIE 2015)

Essa nova tendência educacional preocupa-se com o desenvolvimento do ensino e com a aprendizagem dos alunos, ou seja, com a qualidade dos ambientes que têm sido oferecidos. De acordo com Behar (2010), avaliar é um ato que implica na definição de diversos critérios que devem ser estabelecidos pelo professor. Seja de forma presencial ou à distância, entende-se que a avaliação deve apoiar e incentivar o processo de construção do conhecimento, e não apenas servir como processo classificatório.

Tal processo tem sido facilitado pelas tecnologias existentes e, diante deste cenário, surgem alguns questionamentos quando é pensado no quanto a utilização destes ambientes virtuais são eficazes no propósito inicial, de que maneira é possível medir se os alunos conseguem aprender e se esse conhecimento construído tem mais qualidade quando utilizado o suporte computacional.

O presente trabalho tem o objetivo de apresentar uma proposta para investigar a efetividade do processo de ensino-aprendizado de alunos ao utilizarem os ambientes virtuais Sim-Colmeia e Educ-MAS GA através do modelo de avaliação proposto. Inicialmente, o artigo aborda os principais trabalhos de avaliação de ambientes virtuais. Em seguida, cada ambiente é descrito, pontuando detalhes importantes de cada ferramenta. No final, a metodologia de trabalho utilizada é mostrada, bem como, a conclusão do trabalho.

\section{Avaliação da aprendizagem em ambientes virtuais}

A avaliação do aprendizado dos alunos é uma tarefa complexa, cuja finalidade é analisar se os objetivos educacionais foram atingidos e a eficácia da prática pedagógica [David et al 2007]. A avaliação não se restringe apenas à aprendizagem discente: outros fatores podem influenciar no decorrer deste processo [Souza et al 2013].

Diferenciando-se da instrumentação convencional das avaliações formativas, um processo de avaliação unindo o ambiente computacional aos elementos lúdicos presentes nos jogos computacionais foi introduzido por Araujo (2013). Essa proposta de avaliação formativa da aprendizagem em jogos digitais busca discutir soluções para o uso efetivo dessa avaliação no ambiente de aprendizagem através de um framework para sistematizar o processo de avaliação de modo que respostas, competências e habilidades dos alunos são coletadas e analisadas para subsidiar as ações pedagógicas do professor.

$\mathrm{Na}$ busca da qualidade no processo de ensino e aprendizagem, métodos avaliativos têm sido cada vez mais aperfeiçoados. Venancio (2013), ressalta a importância da avaliação formativa com a implementação do protótipo de um sistema interativo de gestão da avaliação processual baseado em competências. A ferramenta aponta aos educadores a direção a seguir diante da dificuldade de aprendizagem, detectada através do sistema.

Na literatura são propostos alguns modelos de avaliação [Silva e Lima 2014], [Savi et al 2010], [Kirkpatrick 1994] que permitem verificar a efetividade da aprendizagem com a utilização dos ambientes virtuais.

Um elemento considerado essencial em qualquer sistema educacional é a motivação para aprender [Savi et al 2010]. Os indivíduos tem diferentes capacidades e isto torna difícil o processo de motivação que consiga modificar o comportamento dos alunos. Por isso o modelo ARCS tem como objetivo empregar estratégias motivacionais 


\section{CBIE-LACLO 2015}

Anais dos Workshops do IV Congresso Brasileiro de Informática na Educação (CBIE 2015)

no projeto de materiais instrucionais [Savi et al 2010]. ARCS se baseia em quatro categorias de estratégias importantes para se obter a motivação dos alunos no processo de aprendizagem: Atenção, Relevância, Confiança e Satisfação [Savi et al 2010]. A atenção é um elemento motivacional e também uma condição inicial para aprendizagem.

A Relevância é fundamental, pois apenas atenção e curiosidade não são suficientes para que os alunos fiquem motivados sendo preciso que o conhecimento a ser construído seja consistente com seus objetivos. A Confiança está relacionada à tentativa de criar expectativas positivas aos alunos e propor experiências de sucesso ajudam aos alunos a ganharem confiança. Ao motivar adequadamente, mostrando exemplos de recompensas e satisfação pessoal, os alunos tendem a ter sentimentos positivos com a experiência de aprendizagem [Savi et al 2010]. Em ARCS é proposto um questionário denominado Instructional Materials Motivational Scale (IMMS) que deve ser aplicado após a utilização do material educacional por parte dos alunos.

O modelo de avaliação Kirkpatrick (1996) é um modelo de treinamento composto por quatro níveis. Os quatro níveis avaliam (i) a Reação que mede a satisfação e o valor percebido do treinamento pelos participantes; (ii) Aprendizagem que mede o aumento de, novos conhecimentos ou habilidades; (iii) Comportamento que mede a mudança de comportamento em consequência ao conhecimento adquirido. (iv) Resultados que mede efeitos alcançados com treinamento de uma forma geral [Savi et al 2010]. O modelo proposto por Savi (2011) para avaliação de jogos educacionais realiza as avaliações por meio da percepção dos alunos a respeito dos níveis de motivação, experiência do usuário e aprendizagem promovidos por um jogo. Segundo ele, avaliar a reação dos alunos consiste em verificar o quanto eles gostaram de uma ação instrucional e complementa ainda, indicando que "avaliar a reação é o mesmo que medir os sentimentos dos participantes". O modelo de Savi (2011) propõe o uso de um questionário para avaliar escalas. A escala é composta por um total de 29 itens fixos divididos em 3 dimensões (ou sub-escalas) juntamente com uma questão sobre os conteúdos abordados que é especifica para cada jogo. Cada item do questionário possui o formato de resposta que diz respeito ao grau de discordância ou concordância em uma escala com valores entre -2 até +2 . Os respondentes mostram se concordam ou discordam da afirmação quando contribuem com um valor.

Com a preocupação na melhoria do processo de ensino e aprendizagem é necessário que ambientes educacionais sejam avaliados com muito critério. Isso requer aprimoramentos dos métodos de avaliação já existentes e a análise dos resultados para que novas investigações sejam propostas. O modelo de Kirkpatrick (1996) possui níveis de avaliação, porém não tem um formato rígido dos parâmetros que são propostos para serem avaliados no processo de ensino e aprendizagem. Já o modelo de ARCS, possui foco na interação dos alunos com os materiais e ambientes de aprendizagem preocupando-se com a motivação dos alunos. Pode ser aplicado em qualquer situação de aprendizagem já que este processo envolve motivação.

No modelo proposto por Savi (2011), foi usado o modelo ARCS para apoiar o modelo de Kirkpatrick (1996). O modelo ARCS foi utilizado para sustentar a reação do primeiro nível do modelo Kirkpatrick. As categorias de estratégias do modelo ARCS foram consideradas subcomponentes do constructo reação ao jogo educacional. Para Savi (2011), existe no modelo uma relação causal entre os constructos e a qualidade do 


\section{CBIE-LACLO 2015}

Anais dos Workshops do IV Congresso Brasileiro de Informática na Educação (CBIE 2015)

jogo como material educacional, determinada pela reação do aluno ao efeito motivador do jogo, a experiência de jogar e ao ganho de aprendizagem percebido.

\section{Ambientes Virtuais de Aprendizado}

A introdução da computação na educação permite a alunos e professores a análise investigativa de mundos artificiais através ambientes de ensino-aprendizagem [Pereira e Sampaio 2008]. Esses ambientes podem fomentar a discussão dos resultados através das próprias experiências do aluno para construção do seu conhecimento [Lima 2008].

O sistema SIM-Colmeia tem por objetivo disponibilizar um ambiente de simulação para o estudo do comportamento de uma colmeia, utilizando principalmente Inteligência Artificial, como apoio às aulas de Biologia, além de possibilitar interatividade e pode ser usado tanto no ensino médio quanto no ensino fundamental.

Ele simula o crescimento populacional de uma colmeia explorando a relação existente entre a disponibilidade de alimentos (as plantas em floração) e o aumento do número de abelhas da colmeia no ambiente. Possui abrangência nas áreas de Ciências e Biologia. Por meio do Sim-Colmeia o aluno pode manipular alguns parâmetros e perceber os resultados com o decorrer do tempo, interagindo com o mundo virtual e analisando o que acontece partindo de uma hipótese feita por ele mesmo [Figueiredo et al 2012].

O sistema EducMAS GA oferece aprendizagem personalizada no ensino de geometria analítica. Este pode alterar seu comportamento com base no histórico de ações e nas propriedades cognitivas. Com isso, a estratégia de ensino pode ser modificada e o sistema pode se adaptar ao perfil de cada aluno. Atualmente o EducMAS GA é composto por seis agentes, sendo os agentes principais o Agente Coordenador que seleciona a estratégia educacional, o Agente Tutor que agrega as tarefas de um professor, ensinando o aluno através da apresentação dos tópicos, das atividades e da correção dos exercícios e o Agente Afetivo responsável pela interação com o aluno, auxiliando no processo de ensino-aprendizagem [Sousa et al 2012].

\section{Proposta de avaliação de aprendizagem em Ambientes Educacionais}

Nesta seção, é apresentada uma proposta para avaliar a efetividade do uso de ambientes virtuais no processo de ensino-aprendizagem. Pretende-se planejar alguns experimentos e verificar a importância e eficácia da utilização destes ambientes no ensino a que se propõem. A estratégia escolhida neste trabalho é utilizar um modelo de avaliação cujo foco seja a percepção dos alunos antes e depois do contato com os ambientes SimColmeia e Educ-MAS GA, ou seja, realizar avaliações e testes antes e depois da utilização dos ambientes, a fim de avaliar o aumento do conhecimento devido a seu uso.

Assim o processo de avaliação a ser adotado tem as seguintes etapas: (i) Estabelecer uma proposta do Modelo de Avaliação Piloto para o Sim-Colmeia e o EducMAS GA: Nesta etapa foi definida a hipótese e o objetivo que se deseja alcançar a partir do modelo de experimentação piloto e sua contextualização; (ii) Projetar a análise experimental: Nesta etapa é definida a maneira como o experimento deve ser realizado, o ambiente onde será implementado e os participantes; (iii) Aplicar o modelo proposto em turmas de Biologia e Geometria. Preparar o material e os participantes para o experimento. Aplicar a avaliação aos participantes de acordo com o proposto; (iv) 
Analisar os resultados obtidos após a aplicação do modelo. Após conclusão da avaliação, interpretar os resultados obtidos como forma de verificação do processo ocorrido testando estatisticamente se as hipóteses foram atingidas e os objetivos e (v) Reformular o modelo inicial com base nos resultados obtidos.

O modelo piloto de avaliação permite verificar a efetividade da aprendizagem com a utilização dos ambientes virtuais, sendo baseado nos modelos descritos na seção 2. Assim, conforme proposto por Savi (2011), a ideia principal é acoplar a este modelo um módulo baseado no nível 2 de Kirkpatrick, com o intuito de avaliar o ganho de conhecimento por parte dos alunos. Este módulo propõe um modelo de avaliação voltado para ambientes educacionais com a finalidade de evidenciar o impacto de sua utilização no processo de aprendizagem. A Tabela 1 apresenta a comparação entre os modelos de Savi e o modelo da proposta piloto.

Ao realizar o experimento, os ambientes virtuais de aprendizado Sim-Colmeia e Educ-MAS GA serão colocados à disposição de uma turma de Biologia do Ensino Médio e de uma turma de Geometria Analítica na Graduação da Engenharia. Antes e depois da utilização dos ambientes será aplicado um questionário para ser respondido pelos participantes como também um teste para avaliar a aprendizagem desses ambientes. Análise estatística dos dados dos experimentos será realizada e com base nesta será definido o modelo de avaliação de ambientes educacionais do projeto de Ambientes Virtuais Educacionais.

\section{Tabela 1. Comparação entre modelo de Savi (2011) e modelo de avaliação piloto}

\begin{tabular}{|c|c|}
\hline Modelo Savi & Modelo da Proposta Piloto \\
\hline $\begin{array}{c}\checkmark \quad \text { Avaliações baseadas na percepção dos } \\
\text { alunos (Nivel } 1 \text { Kirkpatrick) } \\
\text { Avalia os alunos após o treinamento }\end{array}$ & $\begin{array}{c}\checkmark \text { Avaliações baseadas na aprendizagem dos } \\
\text { alunos (Nivel } 2 \text { Kirkpatrick) } \\
\text { Avalia os alunos antes e após o treinamento }\end{array}$ \\
\hline Avaliação do nível de motivação dos jogos & $\begin{array}{c}\text { Avaliação do nível de motivação dos } \\
\text { ambientes educacionais }\end{array}$ \\
\hline $\begin{array}{l}\text { Avaliação da experiência do usuário } \\
\text { com os jogos }\end{array}$ & $\begin{array}{l}\text { Avaliação da experiência do usuário } \\
\text { com os ambientes educacionais }\end{array}$ \\
\hline $\begin{array}{l}\text { Avaliação da aprendizagem } \\
\text { proporcionada com os jogos }\end{array}$ & $\begin{array}{l}\text { Avaliação da aprendizagem proporcionada com } \\
\text { os ambientes educacionais }\end{array}$ \\
\hline
\end{tabular}

\section{Conclusões}

É importante ressaltar que a utilização de ambientes virtuais educacionais para auxiliar o processo de ensino e aprendizagem tem se tornado cada vez mais frequente por professores que desejam melhorar suas práticas. Assim, tais ambientes podem ser vistos como métodos alternativos de educação visto que os alunos conseguem participar e aprender ao mesmo tempo.

Com isso, a avaliação de ambientes educacionais é vista como ponto importante, vez que se torna necessário entender quais as vantagens de se utilizar este recurso e entender de que maneira podemos melhorar as práticas educacionais já existentes. Portanto este trabalho foi elaborado com o intuito de, além de avaliar o aprendizado dos alunos, demonstrar o quão significante é o uso dessas ferramentas no ensino. Após a aplicação do modelo proposto, ainda existirá diversas outras maneiras de explorarmos a avaliação da aprendizagem já que os alunos são incitados a desenvolver suas ideias e pensar sobre os testes realizados. Assim, deve-se entender que é fundamental que se 


\section{CBIE-LACLO 2015}

Anais dos Workshops do IV Congresso Brasileiro de Informática na Educação (CBIE 2015)

avalie a eficiência dos ambientes educacionais para que estes contribuam de maneira significativa no processo de ensino e aprendizagem.

\section{Referências Bibliográficas}

ARAUJO, G.G., ARANHA, E.H.S. "Avaliação formativa da aprendizagem com instrumentação em Jogos digitais: Proposta de um framework conceitual”. Anais do II Congresso Brasileiro de Informática na Educação (CBIE 2013).

BEHAR, P.A., RIBEIRO, A.C.R., LONGARAY, A.N.C, BERNARDI, M., SILVA, K.K.A. "AVALEAD: um objeto de aprendizagem sobre avaliação em Educação à Distância”. Anais Simpósio Brasileiro de Informática na Educação, João Pessoa, PB, 2010.

DAVID, P.B., PEQUENO, M.C., da SILVA, A., SOUSA, C.F., JÚNIOR, G.S.V., de CASTRO FILHO, J.A., VENTURA, P.P.B. E MAIA, S. "Avaliação da aprendizagem em educação a distância numa perspectiva sócio-interacionista". Anais do XVIII Simpósio Brasileiro de Informática na Educação,2007.

FIGUEIREDO, J.E.M., SILVA, A.S., WERNECK, V.M., COSTA, R.M.E.M. "SimColmeia: Ambiente de simulação da dinâmica populacional de uma colmeia para o ensino de Biologia". Anais do XXII SBIE 2011.

KIRKPATRICK, D. L. (1996). Techniques for evaluating training programs. In; Classic writings on instructional technology, 1(192), 119.

PEREIRA, A.S.T. e SAMPAIO, F.F. "AVITAE: desenvolvimento de um ambiente de modelagem computacional para o ensino da Biologia”. Ciência \& Cognição. São Paulo, v.13.

SAVI, R. Avaliação de jogos voltados para a disseminação do conhecimento. Tese (Doutorado em Engenharia e Gestão do Conhecimento) - Universidade Federal de Santa Catarina, 2011.

SAVI, R.C.S., WANGENHEIM, C.G.V., ULBRICHT,V., VANZIN, T. "Proposta de um Modelo de Avaliação de Jogos Educacionais". RENOTE. Revista Novas Tecnologias na Educação, v. 8, n. 3, p. 1-10, 2010.

SILVA, G.A., LIMA, F. (2014) "Proposta de Aplicação e Avaliação de Jogos Educacionais para o Ensino de Computação no Ensino Fundamental”, em: Workshop da Pós-Graduação em Computação 2014, Brasil.

SOUSA, R.C.S., PERCÚ, G.L., PINTO, P.E.D., FILHO, O.B., WERNECK, V.M.B. “Avaliação Diagnóstica Fuzzy no Educ-MAS GA”. Anais do II Congresso Brasileiro de Sistemas Fuzzy 2012.

SOUZA, T.I.A., FRANCO, A.O.R., SILVA, T.E.V., VASCONCELOS, F.H.L. "Avaliando o Desempenho discente em um AVA: Um Estudo de Caso Utilizando Estatística Multivariada”. Anais do II Congresso Brasileiro de Informática na Educação (CBIE 2013).

VENANCIO, V., LOPES, R.D. "Avaliação da Aprendizagem em Sistemas Interativos: uma revisão comparativa focada no SBIE, WIE e WAvalia". Anais do SBIE 2013. 